\title{
Dry eye symptoms among diabetics: an exploratory study on knowledge, attitude, and practice
}

\author{
Zubaida Sirang1, Tanveer Anjum Chaudhry ${ }^{2}$ \\ 'Section of Ophthalmology, Mater University Hospital, Dublin, Ireland \\ ${ }^{2}$ Section of Ophthalmology, South City Hospital, Karachi, Pakistan
}

\begin{abstract}
BACKGROUND: The objective of the study was to explore the awareness of dry eye symptoms among diabetics attending a tertiary care hospital in Karachi.

MATERIAL AND METHODS: Twenty-five diabetic patients aged $\geq 40$ years were selected from the diabetic clinic of the Aga Khan University Hospital Karachi using convenience sampling. Our primary focus was to determine whether diabetics knew that they were at high risk of developing dry eye symptoms. Participants were also asked both openand closed-ended questions to explore their attitude and practices in relation to dry eye symptoms such as burning, stinging, grittiness, and discomfort.
\end{abstract}

RESULTS: None of the 25 diabetics we interviewed knew that diabetics as compared to their non-diabetic counterparts were at higher risk of developing dry eye syndrome. They did not know about the signs, symptoms, and complications of dry eyes. Twelve participants had symptoms of dry eyes. Among these, five reported washing their eyes with cold water, four reported using no remedy, and one reported using surma. No one reported having consulted an eye doctor.

CONCLUSION: Our study concludes that diabetics did not know that they had an increased risk of developing dry eye symptoms compared with their non-diabetic counterparts. Population-based research is needed to assess dry eye related risk awareness and control measures among diabetics.

KEY WORDS: dry eye disease; diabetics; ocular complications with diabetes

Ophthalmol J 2019; Vol. 4, 105-108

\section{INTRODUCTION}

Dry eye symptoms (burning, stinging, grittiness, and discomfort) affect a significant percentage of the population worldwide, especially people aged 40 years or older [1]. The extent of the problem is well-documented in developed countries such as the United States, where an estimated 25-30 million people have dry eye symptoms $[2,3]$. However, the extent of the problem is not precisely known in South East Asian countries, including Pakistan.
Dry eye symptoms can occur because of a defect in one or more components of the lacrimal functional unit, which comprises the cornea, conjunctiva and meibomian glands, lacrimal glands, the lids, and the associated afferent and efferent nerves [4].

There is plenty of evidence to suggest that diabetics are at a higher risk of getting dry eye symptoms compared with their non-diabetic counterparts $[5,6]$. 
The Canadian Dry Eye Epidemiology Study found that one out of every three people with diabetes had dry eye symptoms [7].

The true prevalence of dry eye symptoms among diabetics can be even higher than this because many diabetics do not know that they have the symptoms because of reduced ocular sensitivity. It is important that diabetics are aware that they are a high-risk group for dry eyes and its complications.

Pakistan has a population of more than 166 million, and more than $10 \%$ of its adult population has diabetes [8].

In this qualitative study, we explored the level of awareness of dry eye symptoms among a small group of diabetics in Karachi, Pakistan.

\section{MATERIAL AND METHODS}

This was a qualitative study. Participants for the study were recruited from the Diabetes Clinic at the Aga Khan University Hospital during April-May 2017. We used convenience sampling to choose the diabetic patients. Recruitment was stopped after the $25^{\text {th }}$ participant, when we felt that we were not getting any new information. Informed consent was obtained from the subjects. We collected data on age, gender, educational status, duration, and type of diabetes. Our primary focus was to assess if diabetics knew that they were at a high risk of developing eyes diseases. Selected individuals were asked the following open-ended request: "Name eye conditions one can have because of diabetes". We also wanted to determine what proportion of them had dry eye symptoms, which was assessed using a validated questionnaire [3].

The questionnaire included questions about the presence of the following six symptoms i.e.:

- feeling of dryness in eyes;

- gritty or sandy sensation in eyes;

- burning sensation in eyes;

- eyes being red;

- crusting on eye lashes;

- eyes stuck shut in the morning.

A five-point Likert scale was used to measure the frequency of symptoms, with $0,1,2,3$, and 4 representing never, rarely, sometimes, often, or all the time, respectively. An individual scoring $\geq 7$ on the $0-24$ scale is labelled as a case of dry eyes. We also asked the selected subjects what they did routinely or would do to manage such dry eye symptoms. The questionnaire was administered in Urdu by one of us (ZS).

\section{RESULTS}

A total of 25 diabetic patients were invited to participate in the study; all responded. The study participants were aged 40 to 69 years [mean $( \pm S D)$ age $=57 \pm 8] ; 15$ were women. (Tab. I). None of the subjects mentioned that diabetics, compared with their non-diabetic counterparts, had a higher risk of developing dry eye symptoms.

Twelve participants had dry eye symptoms. To relieve these symptoms, the primary practice adopted by the majority of them was washing their eyes with cold water, using Aswad Surma (kohl), and avoiding watching TV and excessive use of computer.

Among the 13 asymptomatic patients, seven were of the view that they would not take any relief measure if they developed symptoms of dry eyes. Others reported they would wash their eyes with cold water, use Surma, avoid computer use and dusty environments, and consult a doctor (Tab. II). When the participants were asked to name the ocular complications of diabetes, they mentioned cataract, glaucoma, and retinal problems, but none-in-

Table I. Characteristics of the study participants ( $n=25)$

\begin{tabular}{|l|c|c|}
\hline Characteristic & No. & $\%$ \\
\hline Age group (years) & & \\
\hline $40-49$ & 4 & 16 \\
\hline $50-59$ & 12 & 36 \\
\hline$\geq 60$ & & \\
\hline Sex & 10 & 40 \\
\hline Male & 15 & 60 \\
\hline Female & & \\
\hline Education & 2 & 8 \\
\hline None & 4 & 16 \\
\hline Middle & 8 & 32 \\
\hline Matric & 1 & 4 \\
\hline Higher secondary & 7 & 28 \\
\hline Graduation & 3 & 12 \\
\hline Postgraduation & & \\
\hline Duration of diabetes (years) & 3 & 12 \\
\hline$\leq 4$ & 13 & 52 \\
\hline $5-10$ & 9 & 36 \\
\hline$>10$ & & \\
\hline Type of diabetes & 4 & 16 \\
\hline Type 1 & & 84 \\
\hline Type 2 & & \\
\hline
\end{tabular}




\begin{tabular}{|l|c|c|c|c|}
\hline Table II. Current/intended primary practice for reliving dry eye symptoms by the study subjects & $\begin{array}{c}\text { (n }=25) \\
\text { Individuals with symptoms } \\
\text { of dry eyes }\end{array}$ & $\begin{array}{c}\text { Individuals without symptoms } \\
\text { of dry eyes }\end{array}$ \\
\hline Primary practice & No. & $\%$ & No. & $\%$ \\
\hline Wash with cold water & 5 & 20 & 2 & 8 \\
\hline Don't do anything & 4 & 16 & 7 & 28 \\
\hline Use kohl (Surma) & 1 & 4 & 1 & 4 \\
\hline Avoid watching TV & 1 & 4 & 2 & 8 \\
\hline Use eye drops without going to the doctor & 1 & 4 & - & - \\
\hline Go to an eye specialist & - & - & 1 & 4 \\
\hline Total & 12 & 48 & 13 & 52 \\
\hline
\end{tabular}

Table III. Adverse effects of diabetes on eyes, as reported by the subjects $(n=25)$

\begin{tabular}{|l|c|c|}
\hline Adverse effects & No. & $\% *$ \\
\hline Glaucoma & 20 & 80 \\
\hline Cataract & 15 & 60 \\
\hline Retinal problem & 5 & 20 \\
\hline Don't know & 5 & 20 \\
\hline
\end{tabular}

*Participants could mention more than one adverse effect

cluding the symptomatic ones-mentioned dry eyes (Tab. III).

\section{DISCUSSION}

The diabetics we interviewed were not aware of dry eye symptoms as a complication of diabetes. None of them reported that diabetics compared with their non-diabetic counterparts were at a higher risk of developing dry eyes, although they were aware of other complications, such as cataract, glaucoma, and retinal problems. This finding suggests that people are less likely to be aware of non-sight-threatening conditions than sight-threatening ones such as cataract and glaucoma. Although dry eye is a non-sight-threatening condition, it can interfere with quality of life.

Studies have indicated that the prevalence of dry eyes is as high as $54 \%$ in diabetics [9].

The aetiology of dry eyes among diabetics is not entirely clear. One of the reasons could be the fact that corneal epithelial abnormalities and lacrimal function unit dysfunction can occur in diabetics $[10,11]$.

The diabetics with high glycated haemoglobin $\left(\mathrm{HbA}_{1 \mathrm{c}}\right)$ are said to be more at risk of corneal epithelial dysfunction. Diabetic neuropathy and low immunity may also play a role in the aetiology of dry eyes in diabetics [11]. Corneal complications, i.e. superficial punctate keratopathy, trophic corneal ulcers, persistent epithelial defects, and recurrent corneal erosions can occur both with dry eye disease and hyperglycaemia [11]. The symptoms of dry eyes are more severe in patients with poorly controlled diabetes [12].

It is important that diabetics are aware of dry eye as a complication of this disease from the perspective of prevention.

If they have the appropriate awareness, they will benefit by avoiding unnecessary/excessive exposure to sunlight/high temperatures, wind, air pollution, contact lenses, computers and TVs, and other risk factors. They can seek treatment early and avoid the complications that can occur with chronic dry eye disease, including corneal scarring and visual impairment [11].

Public awareness programmes about the sight-threatening complications of diabetes, such as diabetic retinopathy, cataract, and glaucoma should also include dry eyes and other non-sight-threatening conditions. Targeted awareness can also be channelled through diabetic clinics across the country. Examination for dry eyes should be part of the ocular examination of diabetics.

This was an exploratory study using a small convenience sampling. There is a need to conduct a large-scale study involving a representative sample of diabetics and/or other common high-risk groups and using both self-rated and objective methods of assessment.

\section{CONCLUSION}

This small exploratory study calls for increasing public awareness of dry eye symptoms as a compli- 
cation of diabetes. Both general and targeted awareness programmes on diabetes-related eye diseases should also include dry eye disease.

\section{STATEMENT OF COMPETING INTERESTS}

The authors declare no conflict of interests.

\section{REFERENCES}

1. Brewitt H, Sistani F. Dry eye disease: the scale of the problem. Surv Ophthalmol. 2001; 45 Suppl 2: S199-S202, doi: 10.1016/s00396257(00)00202-2, indexed in Pubmed: 11587143.

2. Schaumberg DA, Sullivan DA, Buring JE, et al. Prevalence of dry eye syndrome among US women. Am J Ophthalmol. 2003; 136(2): 318-326, doi: 10.1016/s0002-9394(03)00218-6, indexed in Pubmed: 12888056.

3. Schein 0, Munoz B, Tielsch J, et al. Prevalence of dry eye among the elderly. Am J Ophthalmol. 1997; 124(6): 723-728, doi: 10.1016/ s0002-9394(14)71688-5, indexed in Pubmed: 9402817.

4. Stern ME, Beuerman RW, Fox Rl, et al. The pathology of dry eye: the interaction between the ocular surface and lacrimal glands. Cornea. 1998; 17(6): 584-589, doi: 10.1097/00003226-199811000-00002, indexed in Pubmed: 9820935.
5. Kaiserman I, Kaiserman N, Nakar $S$, et al. Dry eye in diabetic patients. Am J Ophthalmol. 2005; 139(3): 498-503, doi: 10.1016/j. ajo.2004.10.022, indexed in Pubmed: 15767060.

6. Moss SE, Klein R, Klein BE. Prevalence of and risk factors for dry eye syndrome. Arch Ophthalmol. 2000; 118(9): 1264-1268, doi: 10.1001/ archopht.118.9.1264, indexed in Pubmed: 10980773.

7. Caffery BE, Richter D, Simpson T, et al. CANDEES. The Canadian Dry Eye Epidemiology Study. Adv Exp Med Biol. 1998; 438: 805-806, indexed in Pubmed: 9634970.

8. Shera AS, Jawad F, Maqsood A. Prevalence of diabetes in Pakistan. Diabetes Res Clin Pract. 2007; 76(2): 219-222, doi: 10.1016/j.diabres.2006.08.011, indexed in Pubmed: 17005289

9. Manaviat M, Rashidi M, Afkhami-Ardekani M, et al. Prevalence of dry eye syndrome and diabetic retinopathy in type 2 diabetic patients. BMC Ophthalmology. 2008; 8(1), doi: 10.1186/1471-2415-8-10.

10. Gekka M, Miyata K, Nagai Y, et al. Corneal epithelial barrier function in diabetic patients. Cornea. 2004; 23(1): 35-37, doi: 10.1097/00003226200401000-00006, indexed in Pubmed: 14701955.

11. Zhang $X$, Zhao L, Deng S, et al. Dry Eye Syndrome in Patients with Diabetes Mellitus: Prevalence, Etiology, and Clinical Characteristics. J Ophthalmol. 2016; 2016: 8201053, doi: 10.1155/2016/8201053, indexed in Pubmed: 27213053.

12. Ozdemir M, Buyukbese MA, Cetinkaya A, et al. Risk factors for ocular surface disorders in patients with diabetes mellitus. Diabetes Res Clin Pract. 2003; 59(3): 195-199, doi: 10.1016/s0168-8227(02)00244-9, indexed in Pubmed: 12590016. 